\title{
DEPRESSION AND ALCOHOL USE IN UNDERGRADUATE MEDICAL STUDENTS IN A PRIVATE MEDICAL COLLEGE
}

Meesha Haorongbam¹, Sathyanarayana M. T2, Dhanashree Akshatha H. S3

1Junior Resident, Department of Psychiatry, Sri Siddhartha Medical College, Tumkur, Karnataka.

2 Professor and HOD, Department of Psychiatry, Sri Siddhartha Medical College, Tumkur, Karnataka.

${ }^{3}$ Senior Resident, Department of Psychiatry, Sri Siddhartha Medical College, Tumkur, Karnataka.

\section{ABSTRACT}

\section{BACKGROUND}

Depression can significantly hamper academic performance and can also lead to substance use and abuse. The various causes leading to depression in medical students along with the multiple factors for alcohol use, needs to be assessed in view of rising levels of psychiatric morbidity.

Aim- To study the relationship between depression and alcohol use among undergraduate medical students.

\section{MATERIALS AND METHODS}

A cross-sectional study was done on 428 undergraduate students in a private medical college in Karnataka. They were assessed using a semi-structured proforma, Beck Depression Inventory and Alcohol Use Disorders Identification Test.

\section{RESULTS}

Majority of the students were between 17 - 20 years, females, studying in second year, had never failed earlier, stayed in hostel with roommate (s), were not in a relationship, $13.6 \%$ of the students had moderate depression, $8.4 \%$ had severe depression and 2.8\% had extreme depression. $49.1 \%$ had consumed alcohol at least once. $15.7 \%$ had hazardous level of drinking, while $3.7 \%$ had dependence level of drinking. Common stressors were - need to do well academically and vast amount of content to be learned. Coping measures used by the students were using TV/ internet/ music to relax and seeking out friends for conversation and support.

\section{CONCLUSION}

Depression and alcohol use are highly prevalent in undergraduate medical students. Many of them reported stressors in the academic, relationship and adjustment domains and used various coping strategies to deal with them. Since there is proof that depression or alcohol use during undergraduate medical training foresees future predicaments in physicians, effective measures need to be taken up.

\section{KEYWORDS}

Undergraduate Medical Students, Depression, Alcohol Use.

HOW TO CITE THIS ARTICLE: Haorongbam M, Sathyanarayana MT, Akshatha DHS. Depression and alcohol use in undergraduate medical students in a private medical college. J. Evolution Med. Dent. Sci. 2018;7(12):1473-1479, DOI: 10.14260/jemds/2018/334

\section{BACKGROUND}

The Medical Council of India stipulates four and a half years of undergraduate (UG) medical training followed by a year of compulsory rotating internship. Throughout the course of study, the medical students unavoidably deal with various demanding situations which can have an impact on their mental health. Depression is the second most common mental health problem among medical students.[1] Medical trainees have higher rates of suicidal ideation and suicide attempts compared to the general population. [2] Alarmingly, on an average one physician commits suicide every day in the US.[3] There has also been a surge in the number of suicides in medical students in India in the past few years, which has left many wondering about the reasons for the drastic steps taken by the future healers of the society. $[4,5,6,7]$

'Financial or Other Competing Interest': None.

Submission 06-02-2018, Peer Review 01-03-2018,

Acceptance 09-03-2018, Published 19-03-2018.

Corresponding Author:

Sathyanarayana M. T,

Department of Psychiatry, Sri Siddhartha Medical College,

B. H. Road, Agalakote, Tumkur-572107, Karnataka.

E-mail: sanamathi23@gmail.com

DOI: $10.14260 /$ jemds $/ 2018 / 334$
Few medical students with depression actually seek out treatment; when asked for reasons, they cite lack of time (48\%), lack of confidentiality (37\%), stigma (30\%), cost (28\%) and fear of documentation on their records (24\%). ${ }^{[8]}$

Depression among medical students also places a greater risk for problems such as substance use or abuse. This assumes a special significance among the medical students who are the future medical practitioners and have a potential role in treating and counselling the patients of substance use disorders. A study by Seshadri $S^{[9]}$ reported that the prevalence of alcohol use among UGs was 9\%. Sohail N[10] observed a general acceptance among UGs that alcohol could provide positive reinforcement. In view of the fact that mental health conditions such as depression and alcohol use among doctors can directly hamper patient care, this study was undertaken.

The aim of this study was to study the relationship between stress and depression and alcohol use among undergraduate medical students. The objectives were: (1) To screen for the prevalence of depression and alcohol use among undergraduate medical students, (2) To establish the relationship between the prevalence of depression and alcohol use. 


\section{MATERIALS AND METHODS}

This was a cross-sectional study conducted in a private medical college in South India between November 2015 and April 2016. All undergraduate students were included in the study, while all interns and those refusing to give consent were excluded. A total of 428 students fulfilling the inclusion criteria participated in the study. Written informed consent was taken from the participants after explaining the nature of the study. To ensure anonymity, students were asked not to mention their name anywhere in the questionnaire.

\section{Study Tools Used}

1. A self-administered, semi-structured proforma questionnaire compiled by the authors pertaining to students' demographic variables and 12 closed and open ended questions.

2. Beck Depression Inventory (BDI)[11]: It is used for measuring the severity of depression and consists of 21 groups of statements. Participants are asked to grade from $0-3$ on how they have been feeling during the previous two weeks. Based on the total score it is divided into: Mild mood disturbance (11 - 16), Borderline clinical depression (17 - 20), Moderate depression (21 - 30), Severe depression (31 - 40) and Extreme depression (> 40).

3. Alcohol Use Disorder Identification Test (AUDIT)[12]: AUDIT helps to identify alcohol dependence and some specific consequences of harmful drinking. There are 10 questions and a maximum score of 40 . Total scores between 8 and 19 indicate hazardous drinking and scores $>20$ indicate dependence level.

\section{Statistical Analyses}

Data was analysed using SPSS 20 (IBM). Proportion was compared using Chi-square test.

\begin{tabular}{|c|c|c|c|}
\hline & \multirow{2}{*}{\multicolumn{2}{|c|}{ Total $(\mathrm{N}=428)$}} \\
\hline & & & \\
\hline & & $\mathrm{n}$ & $\%$ \\
\hline \multirow{4}{*}{ Age } & $17-20$ yrs. & 231 & 54.0 \\
\hline & $21-23$ yrs. & 173 & 40.4 \\
\hline & $24-26$ yrs. & 24 & 5.6 \\
\hline & Mean \pm SD & \multicolumn{2}{|c|}{$20.4 \pm 1.803$} \\
\hline \multirow{2}{*}{ Gender } & Male & 212 & 49.5 \\
\hline & Female & 216 & 50.5 \\
\hline \multirow{4}{*}{ Year } & First & 102 & 23.8 \\
\hline & Second & 131 & 30.6 \\
\hline & Third & 106 & 24.8 \\
\hline & Final & 89 & 20.8 \\
\hline \multirow{6}{*}{$\begin{array}{l}\text { Failures in the } \\
\text { Previous Terms }\end{array}$} & Never Failed & 253 & 59.1 \\
\hline & Failed Once & 135 & 31.5 \\
\hline & Failed Twice & 29 & 6.8 \\
\hline & Failed Thrice & 9 & 2.1 \\
\hline & Failed Four Times & 1 & .2 \\
\hline & Failed Five Times & 1 & .2 \\
\hline \multirow{5}{*}{$\begin{array}{c}\text { Living } \\
\text { Arrangement }\end{array}$} & Hostel- Alone & 46 & 10.7 \\
\hline & Hostel with Roommate & 274 & 64.0 \\
\hline & Rent- Alone & 26 & 6.1 \\
\hline & Rent with Roommate & 24 & 5.6 \\
\hline & With Parents & 58 & 13.6 \\
\hline \multirow{2}{*}{$\begin{array}{c}\text { Relationship } \\
\text { Status }\end{array}$} & In a Relationship & 108 & 25.2 \\
\hline & Others & 320 & 74.8 \\
\hline \multirow{2}{*}{ Domicile } & Urban & 368 & 86.0 \\
\hline & Rural & 60 & 14.0 \\
\hline
\end{tabular}

\begin{tabular}{|c|c|c|c|}
\hline $\begin{array}{c}\text { Selection/ Choice } \\
\text { of Course }\end{array}$ & Forced by Parents & 50 & 11.7 \\
\cline { 2 - 4 } & Own Choice & 378 & 88.3 \\
\hline Table 1. Socio-Demographic Details of the Students \\
(N=428)
\end{tabular}

\begin{tabular}{|c|c|c|}
\hline BDI & Frequency & Percent \\
\hline No depression & 183 & 42.8 \\
\hline Mild mood disturbance & 90 & 21.0 \\
\hline Borderline clinical depression & 49 & 11.4 \\
\hline Moderate depression & 58 & 13.6 \\
\hline Severe depression & 36 & 8.4 \\
\hline Extreme depression & 12 & 2.8 \\
\hline Total & 428 & $100.0 \%$ \\
\hline
\end{tabular}

*BDI- Beck Depression Inventory. Depression is categorised into: No depression $(\leq 10)$, Mild mood disturbance $(11$ - 16), Borderline clinical depression (17 20), Moderate depression (21 - 30), Severe depression (31 40), Extreme depression (>40).

\begin{tabular}{|c|c|c|}
\hline & \multicolumn{2}{|c|}{$\begin{array}{c}\text { Total } \\
(\mathrm{N}=428)\end{array}$} \\
\hline & $\mathbf{n}$ & $\%$ \\
\hline \multicolumn{3}{|l|}{ Academic Domain } \\
\hline Getting poor marks & 215 & $50.2 \%$ \\
\hline Facing death of the patients & 101 & $23.6 \%$ \\
\hline Unjustified grading process & 177 & $41.4 \%$ \\
\hline Heavy workload & 280 & $65.4 \%$ \\
\hline Lack of recognition for work done & 164 & $38.3 \%$ \\
\hline Lack of guidance from teacher & 111 & $25.9 \%$ \\
\hline Difficulty to answer questions from teachers & 167 & $39.0 \%$ \\
\hline Vast amount of content to be learnt & 303 & $70.8 \%$ \\
\hline Need to do well & 306 & $71.7 \%$ \\
\hline Poor motivation to learn & 140 & $32.7 \%$ \\
\hline $\begin{array}{l}\text { Difficulty in understanding the content of the } \\
\text { course }\end{array}$ & 150 & $35.0 \%$ \\
\hline Others & 40 & $9.3 \%$ \\
\hline \multicolumn{3}{|l|}{ Relationship Domain } \\
\hline $\begin{array}{l}\text { Relationship difficulties with parents/ } \\
\text { friends/significant others }\end{array}$ & 136 & $31.8 \%$ \\
\hline Lack of time for family and friends & 165 & $38.6 \%$ \\
\hline Conflicts with other students & 96 & $22.4 \%$ \\
\hline Others & 54 & $12.6 \%$ \\
\hline \multicolumn{3}{|l|}{ Adjustment Domain } \\
\hline Problems with food & 251 & $58.6 \%$ \\
\hline Parental separation/ bereavement & 117 & $27.3 \%$ \\
\hline Managing transition & 153 & $35.7 \%$ \\
\hline Others & 110 & $25.7 \%$ \\
\hline \multicolumn{3}{|l|}{ Emotions Domain } \\
\hline Loneliness and homesickness & 165 & $38.6 \%$ \\
\hline $\begin{array}{c}\text { Lack of self-confidence or low } \\
\text { self esteem }\end{array}$ & 151 & $35.3 \%$ \\
\hline Feeling of incompetence & 131 & $30.6 \%$ \\
\hline Self injury & 45 & $10.5 \%$ \\
\hline Suicidal thoughts & 39 & $9.1 \%$ \\
\hline Others & 30 & $7.0 \%$ \\
\hline \multicolumn{3}{|l|}{ Personal Management Domain } \\
\hline Worries about self appearance & 141 & $32.9 \%$ \\
\hline Issues around sex and sexuality & 25 & $5.8 \%$ \\
\hline Others & 61 & $14.3 \%$ \\
\hline
\end{tabular}

*Multiple answers. 


\begin{tabular}{|c|c|c|}
\hline & \multicolumn{2}{|c|}{ Total $(\mathrm{N}=428)$} \\
\hline & $\mathbf{n}$ & $\%$ \\
\hline Use internet/ TV/ music to relax & 357 & $84.6 \%$ \\
\hline Use humour to take the edge off & 185 & $44.7 \%$ \\
\hline $\begin{array}{l}\text { Seek out friends for conversation and } \\
\text { support }\end{array}$ & 266 & $63.8 \%$ \\
\hline Maintain a healthy diet & 158 & $36.9 \%$ \\
\hline $\begin{array}{l}\text { Get involved in a hobby or interest that } \\
\text { help me unwind and enjoy myself }\end{array}$ & 229 & $53.5 \%$ \\
\hline $\begin{array}{l}\text { Just ignore the problem and hope it } \\
\text { will go away }\end{array}$ & 216 & $50.5 \%$ \\
\hline Pray or meditate & 151 & $35.3 \%$ \\
\hline Go out shopping & 144 & $33.6 \%$ \\
\hline $\begin{array}{l}\text { Confront the source of depression and } \\
\text { work to change it }\end{array}$ & 161 & $37.6 \%$ \\
\hline Sleep more than usual & 228 & $53.3 \%$ \\
\hline $\begin{array}{c}\text { Get irritable and take it out on those } \\
\text { around }\end{array}$ & 166 & $38.8 \%$ \\
\hline $\begin{array}{l}\text { Withdraw emotionally and just go } \\
\text { through the motions of the day }\end{array}$ & 140 & $32.8 \%$ \\
\hline Seek professional help & 69 & $16.1 \%$ \\
\hline Drink alcohol & 76 & $17.8 \%$ \\
\hline Smoke a cigarette & 55 & $12.9 \%$ \\
\hline Others & 16 & $3.8 \%$ \\
\hline $\begin{array}{l}\text { 4. Table showing the Coping } 1 \\
\text { Students to deal with Depres }\end{array}$ & $N=4$ & by the \\
\hline
\end{tabular}

*Multiple answers.

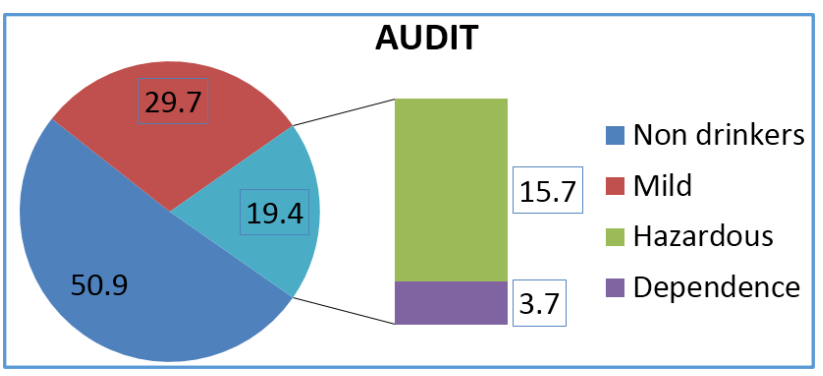

Figure 1. Prevalence of Alcohol Consumption in the Students as assessed by AUDIT (N=428)

*AUDIT- Alcohol Use Disorder Identification Test. Nondrinkers- 0, Mild: 1 - 7, Hazardous: 8 - 19, Dependence: $\geq 20$.

\begin{tabular}{|c|c|c|}
\hline Reason for Initiation & $\mathbf{n}$ & $\mathbf{\%}$ \\
\hline Friend's influence & 65 & $15.2 \%$ \\
\hline Party & 75 & $17.5 \%$ \\
\hline Social gathering & 42 & $9.8 \%$ \\
\hline Curiosity & 84 & $19.6 \%$ \\
\hline Others & 14 & $3.3 \%$ \\
\hline Table 5. Reason for Initiation of Alcohol Consumption \\
$(\mathbf{N}=\mathbf{2 1 0})^{*}$
\end{tabular}

*Multiple answers.

\begin{tabular}{|c|c|c|}
\hline Reason for Drinking & $\mathbf{n}$ & $\mathbf{\%}$ \\
\hline Enjoyment & 135 & $31.5 \%$ \\
\hline To take mind off other issues & 41 & $9.6 \%$ \\
\hline Socialisation & 63 & $14.8 \%$ \\
\hline Table 6. Reason for Drinking (N=210)* \\
\hline
\end{tabular}

*Multiple answers.

\begin{tabular}{|c|c|c|}
\hline Type of Alcohol & $\mathbf{n}$ & $\mathbf{\%}$ \\
\hline Beer & 118 & $27.6 \%$ \\
\hline Whisky & 71 & $16.6 \%$ \\
\hline Rum & 57 & $13.3 \%$ \\
\hline Brandy & 34 & $8.0 \%$ \\
\hline Vodka & 88 & $20.6 \%$ \\
\hline Wine & 55 & $12.9 \%$ \\
\hline Breezer & 70 & $16.4 \%$ \\
\hline Table 7. Type of Alcohol Consumed (N=210) \\
\hline
\end{tabular}

*Multiple answers.

\begin{tabular}{|c|c|c|}
\hline Family Member & $\mathbf{n}$ & $\mathbf{\%}$ \\
\hline Father & 94 & $44.7 \%$ \\
\hline Mother & 8 & $3.8 \%$ \\
\hline Brother & 57 & $27.1 \%$ \\
\hline Sister & 10 & $4.7 \%$ \\
\hline $\begin{array}{c}\text { Table 8. Alcohol Consumption in Family Members of } \\
\text { Student Who Drink (N=210)* }\end{array}$ \\
\hline
\end{tabular}

*Multiple answers.

\begin{tabular}{|c|c|c|}
\hline Family Member & $\mathbf{n}$ & $\mathbf{\%}$ \\
\hline Father & 29 & $13.3 \%$ \\
\hline Mother & 3 & $1.4 \%$ \\
\hline Brother & 15 & $6.9 \%$ \\
\hline Sister & 1 & $0.5 \%$ \\
\hline $\begin{array}{c}\text { Table 9. Alcohol Consumption in Family Members of } \\
\text { Student Who Do Not Drink (N=218)* }\end{array}$ \\
\hline
\end{tabular}

*Multiple answers.

\begin{tabular}{|c|c|c|c|c|c|c|c|c|c|c|c|c|c|c|}
\hline \multirow{3}{*}{ Parameter } & \multirow{3}{*}{ Category } & \multicolumn{12}{|c|}{ Beck Depression Inventory } & \multirow{3}{*}{$\begin{array}{c}P \\
\text { value }\end{array}$} \\
\hline & & \multicolumn{2}{|c|}{$\begin{array}{c}\text { Normal } \\
(\mathrm{N}=183)\end{array}$} & \multicolumn{2}{|c|}{$\begin{array}{c}\text { Mild } \\
(\mathrm{N}=90)\end{array}$} & \multicolumn{2}{|c|}{$\begin{array}{c}\text { Borderline } \\
(\mathrm{N}=49)\end{array}$} & \multicolumn{2}{|c|}{$\begin{array}{c}\text { Moderate } \\
(\mathrm{N}=58)\end{array}$} & \multicolumn{2}{|c|}{$\begin{array}{l}\text { Severe } \\
(\mathrm{N}=36)\end{array}$} & \multicolumn{2}{|c|}{$\begin{array}{c}\text { Extreme } \\
(\mathrm{N}=12)\end{array}$} & \\
\hline & & $\mathrm{n}$ & $\%$ & $\mathrm{n}$ & $\%$ & $\mathrm{n}$ & $\%$ & $\mathrm{n}$ & $\%$ & $\mathrm{n}$ & $\%$ & $\mathrm{n}$ & $\%$ & \\
\hline \multirow{3}{*}{ Age } & $17-20$ yrs. & 103 & $56.3 \%$ & 56 & $62.2 \%$ & 25 & $51.0 \%$ & 25 & $43.1 \%$ & 15 & $41.7 \%$ & 7 & $58.3 \%$ & \multirow{3}{*}{0.081} \\
\hline & $21-23$ yrs. & 74 & $40.4 \%$ & 31 & $34.4 \%$ & 20 & $40.8 \%$ & 25 & $43.1 \%$ & 18 & $50.0 \%$ & 5 & $41.7 \%$ & \\
\hline & $24-26$ yrs. & 6 & $3.3 \%$ & 3 & $3.3 \%$ & 4 & $8.2 \%$ & 8 & $13.8 \%$ & 3 & $8.3 \%$ & 0 & $0.0 \%$ & \\
\hline \multirow{2}{*}{ Gender } & Male & 91 & $49.7 \%$ & 40 & $44.4 \%$ & 20 & $40.8 \%$ & 32 & $55.2 \%$ & 22 & $61.1 \%$ & 7 & $58.3 \%$ & \multirow{2}{*}{0.362} \\
\hline & Female & 92 & $50.3 \%$ & 50 & $55.6 \%$ & 29 & $59.2 \%$ & 26 & $44.8 \%$ & 14 & $38.9 \%$ & 5 & $41.7 \%$ & \\
\hline \multirow{4}{*}{ Year } & First & 47 & $25.7 \%$ & 27 & $30.0 \%$ & 9 & $18.4 \%$ & 11 & $19.0 \%$ & 5 & $13.9 \%$ & 3 & $25.0 \%$ & \multirow{4}{*}{0.452} \\
\hline & Second & 57 & $31.1 \%$ & 22 & $24.4 \%$ & 17 & $34.7 \%$ & 18 & $31.0 \%$ & 13 & $36.1 \%$ & 4 & $33.3 \%$ & \\
\hline & Third & 46 & $25.1 \%$ & 26 & $28.9 \%$ & 11 & $22.4 \%$ & 13 & $22.4 \%$ & 6 & $16.7 \%$ & 4 & $33.3 \%$ & \\
\hline & Final & 33 & $18.0 \%$ & 15 & $16.7 \%$ & 12 & $24.5 \%$ & 16 & $27.6 \%$ & 12 & $33.3 \%$ & 1 & $8.3 \%$ & \\
\hline
\end{tabular}




\begin{tabular}{|c|c|c|c|c|c|c|c|c|c|c|c|c|c|c|}
\hline \multirow{6}{*}{ Failures } & Never failed & 118 & $64.5 \%$ & 59 & $65.6 \%$ & 26 & $53.1 \%$ & 31 & $53.4 \%$ & 14 & $38.9 \%$ & 5 & $41.7 \%$ & \multirow{6}{*}{0.202} \\
\hline & Failed once & 51 & $27.9 \%$ & 25 & $27.8 \%$ & 18 & $36.7 \%$ & 20 & $34.5 \%$ & 16 & $44.4 \%$ & 5 & $41.7 \%$ & \\
\hline & Failed twice & 10 & $5.5 \%$ & 5 & $5.6 \%$ & 4 & $8.2 \%$ & 5 & $8.6 \%$ & 4 & $11.1 \%$ & 1 & $8.3 \%$ & \\
\hline & \begin{tabular}{|l|} 
Failed thrice \\
\end{tabular} & 4 & $2.2 \%$ & 0 & $.0 \%$ & 0 & $0 \%$ & 2 & $3.4 \%$ & 2 & $5.6 \%$ & 1 & $8.3 \%$ & \\
\hline & $\begin{array}{c}\text { Failed four } \\
\text { times }\end{array}$ & 0 & $.0 \%$ & 1 & $1.1 \%$ & 0 & $.0 \%$ & 0 & $.0 \%$ & 0 & $.0 \%$ & 0 & $.0 \%$ & \\
\hline & $\begin{array}{l}\text { Failed five } \\
\text { times }\end{array}$ & 0 & $.0 \%$ & 0 & $.0 \%$ & 1 & $2.0 \%$ & 0 & $.0 \%$ & 0 & $.0 \%$ & 0 & $.0 \%$ & \\
\hline \multirow{5}{*}{ Living } & Hostel- alone & 17 & $9.3 \%$ & 14 & $15.6 \%$ & 8 & $16.3 \%$ & 3 & $5.2 \%$ & 4 & $11.1 \%$ & 0 & $.0 \%$ & \multirow{5}{*}{0.070} \\
\hline & $\begin{array}{l}\text { Hostel with } \\
\text { roommate }\end{array}$ & 113 & $61.7 \%$ & 57 & $63.3 \%$ & 32 & $65.3 \%$ & 42 & $72.4 \%$ & 23 & $63.9 \%$ & 7 & $58.3 \%$ & \\
\hline & Rent-alone & 16 & $8.7 \%$ & 2 & $2.2 \%$ & 1 & $2.0 \%$ & 5 & $8.6 \%$ & 1 & $2.8 \%$ & 1 & $8.3 \%$ & \\
\hline & $\begin{array}{l}\text { Rent with } \\
\text { roommate }\end{array}$ & 4 & $2.2 \%$ & 8 & $8.9 \%$ & 3 & $6.1 \%$ & 3 & $5.2 \%$ & 4 & $11.1 \%$ & 2 & $16.7 \%$ & \\
\hline & With parents & 33 & $18.0 \%$ & 9 & $10.0 \%$ & 5 & $10.2 \%$ & 5 & $8.6 \%$ & 4 & $11.1 \%$ & 2 & $16.7 \%$ & \\
\hline \multirow[t]{2}{*}{ Marital Status } & \begin{tabular}{|c|} 
In \\
relationship
\end{tabular} & 40 & $21.9 \%$ & 25 & $27.8 \%$ & 14 & $28.6 \%$ & 12 & $20.7 \%$ & 15 & $41.7 \%$ & 2 & $16.7 \%$ & \multirow[t]{2}{*}{0.159} \\
\hline & Others & 143 & $78.1 \%$ & 65 & $72.2 \%$ & 35 & $71.4 \%$ & 46 & $79.3 \%$ & 21 & $58.3 \%$ & 10 & $83.3 \%$ & \\
\hline \multirow{2}{*}{ Domicile } & Urban & 159 & $86.9 \%$ & 79 & $87.8 \%$ & 41 & $83.7 \%$ & 47 & $81.0 \%$ & 32 & $88.9 \%$ & 10 & $83.3 \%$ & \multirow{2}{*}{0.838} \\
\hline & Rural & 24 & $13.1 \%$ & 11 & $12.2 \%$ & 8 & $16.3 \%$ & 11 & $19.0 \%$ & 4 & $11.1 \%$ & 2 & $16.7 \%$ & \\
\hline \multirow{2}{*}{$\begin{array}{c}\text { Selection/ Choice } \\
\text { of Course }\end{array}$} & $\begin{array}{l}\text { Forced by } \\
\text { parents }\end{array}$ & 8 & $4.4 \%$ & 15 & $16.7 \%$ & 6 & $12.2 \%$ & 8 & $13.8 \%$ & 11 & $30.6 \%$ & 2 & $16.7 \%$ & \multirow[t]{2}{*}{0.001} \\
\hline & Own choice & 175 & $95.6 \%$ & 75 & $83.3 \%$ & 43 & $87.8 \%$ & 50 & $86.2 \%$ & 25 & $69.4 \%$ & 10 & $83.3 \%$ & \\
\hline \multicolumn{15}{|c|}{ Table 10. BDI Parameters } \\
\hline
\end{tabular}

\begin{tabular}{|c|c|c|c|c|c|c|c|c|c|c|}
\hline \multirow{3}{*}{ Parameter } & \multirow{3}{*}{ Category } & \multicolumn{8}{|c|}{ AUDIT } & \multirow{3}{*}{ P value } \\
\hline & & \multicolumn{2}{|c|}{$\begin{array}{l}\text { Non-Alcoholic } \\
(\mathrm{N}=\mathbf{2 1 8})\end{array}$} & \multicolumn{2}{|c|}{ Mild (N=127) } & \multicolumn{2}{|c|}{$\begin{array}{l}\text { Hazardous } \\
(\mathrm{N}=67)\end{array}$} & \multicolumn{2}{|c|}{$\begin{array}{c}\text { Dependence } \\
(\mathrm{N}=16)\end{array}$} & \\
\hline & & $\mathrm{n}$ & $\%$ & $\mathrm{n}$ & $\%$ & $\mathrm{n}$ & $\%$ & $\mathrm{n}$ & $\%$ & \\
\hline \multirow{3}{*}{ Age } & $17-20$ yrs. & 142 & $65.1 \%$ & 61 & $48.0 \%$ & 23 & $34.3 \%$ & 5 & $31.3 \%$ & \multirow{3}{*}{$<0.001$} \\
\hline & $21-23$ yrs. & 73 & $33.5 \%$ & 57 & $44.9 \%$ & 34 & $50.7 \%$ & 9 & $56.3 \%$ & \\
\hline & $24-26$ yrs. & 3 & $1.4 \%$ & 9 & $7.1 \%$ & 10 & $14.9 \%$ & 2 & $12.5 \%$ & \\
\hline \multirow{2}{*}{ Gender } & Male & 75 & $34.4 \%$ & 72 & $56.7 \%$ & 51 & $76.1 \%$ & 14 & $87.5 \%$ & \multirow{2}{*}{$<0.001$} \\
\hline & Female & 143 & $65.6 \%$ & 55 & $43.3 \%$ & 16 & $23.9 \%$ & 2 & $12.5 \%$ & \\
\hline \multirow{4}{*}{ Year } & First & 64 & $29.4 \%$ & 28 & $22.0 \%$ & 10 & $14.9 \%$ & 0 & $0.0 \%$ & \multirow{4}{*}{$<0.001$} \\
\hline & Second & 68 & $31.2 \%$ & 38 & $29.9 \%$ & 19 & $28.4 \%$ & 6 & $37.5 \%$ & \\
\hline & Third & 53 & $24.3 \%$ & 38 & $29.9 \%$ & 11 & $16.4 \%$ & 4 & $25.0 \%$ & \\
\hline & Final & 33 & $15.1 \%$ & 23 & $18.1 \%$ & 27 & $40.3 \%$ & 6 & $37.5 \%$ & \\
\hline \multirow{6}{*}{ Failures } & Never failed & 152 & $69.7 \%$ & 73 & $57.5 \%$ & 25 & $37.3 \%$ & 3 & $18.8 \%$ & \multirow{6}{*}{$<0.001$} \\
\hline & Failed once & 59 & $27.1 \%$ & 39 & $30.7 \%$ & 28 & $41.8 \%$ & 9 & $56.3 \%$ & \\
\hline & Failed twice & 5 & $2.3 \%$ & 11 & $8.7 \%$ & 11 & $16.4 \%$ & 2 & $12.5 \%$ & \\
\hline & Failed thrice & 2 & $0.9 \%$ & 3 & $2.4 \%$ & 2 & $3.0 \%$ & 2 & $12.5 \%$ & \\
\hline & Failed four times & 0 & $0.0 \%$ & 1 & $0.8 \%$ & 0 & $0.0 \%$ & 0 & $0.0 \%$ & \\
\hline & Failed five times & 0 & $0.0 \%$ & 0 & $0.0 \%$ & 1 & $1.5 \%$ & 0 & $0.0 \%$ & \\
\hline \multirow{5}{*}{ Living Arrangement } & Hostel- alone & 23 & $10.6 \%$ & 14 & $11.0 \%$ & 9 & $13.4 \%$ & 0 & $0.0 \%$ & \multirow{5}{*}{0.241} \\
\hline & $\begin{array}{l}\text { Hostel with } \\
\text { roommate }\end{array}$ & 142 & $65.1 \%$ & 80 & $63.0 \%$ & 42 & $62.7 \%$ & 10 & $62.5 \%$ & \\
\hline & Rent-alone & 8 & $3.7 \%$ & 10 & $7.9 \%$ & 7 & $10.4 \%$ & 1 & $6.3 \%$ & \\
\hline & $\begin{array}{l}\text { Rent with } \\
\text { roommate }\end{array}$ & 12 & $5.5 \%$ & 5 & $3.9 \%$ & 4 & $6.0 \%$ & 3 & $18.8 \%$ & \\
\hline & With parents & 33 & $15.1 \%$ & 18 & $14.2 \%$ & 5 & $7.5 \%$ & 2 & $12.5 \%$ & \\
\hline \multirow{2}{*}{ Relationship Status } & In a relationship & 42 & $19.3 \%$ & 41 & $32.3 \%$ & 21 & $31.3 \%$ & 4 & $25.0 \%$ & \multirow{2}{*}{0.032} \\
\hline & Others & 176 & $80.7 \%$ & 86 & $67.7 \%$ & 46 & $68.7 \%$ & 12 & $75.0 \%$ & \\
\hline \multirow{2}{*}{ Domicile } & Urban & 189 & $86.7 \%$ & 110 & $86.6 \%$ & 58 & $86.6 \%$ & 11 & $68.8 \%$ & \multirow{2}{*}{0.251} \\
\hline & Rural & 29 & $13.3 \%$ & 17 & $13.4 \%$ & 9 & $13.4 \%$ & 5 & $31.3 \%$ & \\
\hline \multirow{2}{*}{$\begin{array}{c}\text { Selection/ Choice of } \\
\text { Course }\end{array}$} & Forced by parents & 23 & $10.6 \%$ & 12 & $9.4 \%$ & 13 & $19.4 \%$ & 2 & $12.5 \%$ & \multirow{2}{*}{0.190} \\
\hline & Own choice & 195 & $89.4 \%$ & 115 & $90.6 \%$ & 54 & $80.6 \%$ & 14 & $87.5 \%$ & \\
\hline
\end{tabular}




\section{DISCUSSION}

In this study, an attempt was made to study the prevalence of depression and alcohol use in undergraduate medical students of a private medical college. We have also attempted to find the relationship between depression and alcohol use.

\section{Depression}

In this study it was found that a total of $36.2 \%$ students had depression, which is higher than that of another similar study where the overall prevalence of depression among the students was $29.78 \% .{ }^{[13]}$ Though it was not statistically significant, the prevalence of severe and extreme depression was higher in males $(61.1 \%$ and $58.3 \%$ respectively) as compared to females ( $38.9 \%$ and $41.7 \%$, respectively). This is similar to a study by Kumar and Jain, which showed a higher prevalence of depression in males.[14] However, another study by Yadav, Gupta and Malhotra found contrasting results, wherein females had higher prevalence of depression than males.[15] Second year students had the highest prevalence of severe (31\%) and extreme (33.3\%) depression. It was also observed that those students who had never failed and those who had failed once had the highest prevalence of extreme depression (7.1\%).

Various studies reported that academic difficulties exert pressure on undergraduate medical students, which cause emotional disturbances in them.[16-20] Similar findings were obtained in this study. Though students reported stressors in every domain, maximum was seen in academic domain where $71.7 \%$ of the students reported that a constant need to do well was distressing them, while $70.8 \%$ reported vast amount of content to be learned and $65.4 \%$ reported heavy workload as the cause for depression.

Relationship difficulties such as lack of time for friends, family and significant others (38.6\%) were also cited as reasons. As many students were studying in a different place with different culture and were staying away from their friends and family for the first time, adjustment problems also proved to be distressing for the students. $58.6 \%$ had problems with food, while $27.3 \%$ were affected by parental separation and $35.7 \%$ reported managing transition as the main stressor. In the personal domain 32.9\% reported worries about their self appearance, while $5.8 \%$ had issues around sex and sexuality. Emotional difficulties such as loneliness or homesickness (38.6\%), lack of self-confidence $(35.3 \%)$ and feeling of incompetence $(30.6 \%)$ were also reported as stressors by the students. Table 3 shows that $10.5 \%$ had indulged in self injurious activities, while $9.1 \%$ had suicidal thoughts. It is quite alarming that students who are being trained as future healers, who have to help others with physical and/or mental problems are harbouring suicidal thoughts. This, in addition to individual torment may unconstructively affect patient care.

\section{Coping}

Students use various coping mechanisms to process stress. Strategies such as problem solving, positive reinterpretation and expression of emotion facilitate student adaptation, which reduces depression and their effects on mental wellbeing and physical health. Studies reported that extracurricular activities involving music and physical exercise have been associated with decreased burnout levels in medical students.[21,22,23] We found that majority of students (84.6\%) use internet/ TV/ music to relax. Some (63.8\%) sought out friends for conversation and support, while $56.3 \%$ tried to focus on the things they can control and accept the things that they cannot. They also used various coping methods such as maintaining a healthy diet (36.9\%), shopping (33.6\%) and alcohol (17.8\%). It was interesting to note that only $16.1 \%$ reported to have sought professional help.

\section{Alcohol}

It was found in our study that $49.1 \%$ students had consumed alcohol at least once in their lives. Age, gender, academic year, failures and relationship status were statistically associated with drinking. Hazardous and dependence level of drinking were seen more in males than females $(76.1 \%$ and $87.5 \%$ respectively), which is similar to findings from other studies.[24,25,26] While hazardous drinking was more prevalent in final year $(40.3 \%)$, dependence was equally present in second and final year. Hazardous (41.8\%) and dependence (56.3\%) level of drinking were seen more in those students who had failed once since joining the course. It was also significantly prevalent in those who were not in a relationship ( $p$ value 0.032). Most of the students started consuming alcohol between the ages of 18 to 20, which was older than the finding by Devi[27] which revealed that most of the medical students started drinking by the age of 16 years. There was however one student who tried alcohol for the first time at age 9. Curiosity (40\%) appears to be the most common reason for initiating alcohol consumption. $64.2 \%$ claimed that they were drinking for enjoyment, while only $19.5 \%$ drank to take their mind off other issues. A similar finding was noticed by Devi[27] and Apoorva.[28]

Similar to a result from a multicentric cross-sectional study[26] done earlier, this study found that the most commonly consumed alcoholic beverage among the students was beer (56.2\%), closely followed by vodka (41.9\%). Among girls vodka was more popular followed by breezer. $36.2 \%$ of those who consumed alcohol had thought about quitting at some point of time citing various reasons such as health issues, pressure from parents, not liking the taste etc. 5.7\% responded that alcohol had affected their concentration, while $9.5 \%$ reported that alcohol had affected their attendance to class with some claiming that alcohol had made them unable to get up in the next morning. This was reported less by girls as compared to boys. Leavy and Alexander[29] recorded that $49 \%$ of medical students had missed morning lectures due to drinking. In the Delk and Meilman study, [30] $56.2 \%$ maintained that they had missed a class as a consequence of their drinking. It is worth mentioning that alcohol might be a convenient scapegoat. It can be speculated that questionnaires which ask about drinking behaviour and then about attendance and concentration may unwittingly provide a link which the student is willing to follow.

A startling $46.2 \%$ claimed that alcohol had reduced depression in their lives. Many females responded that they have tried alcohol, but had given up by the time this study was undertaken. The reasons given by them were: (1) They did not like the taste of alcohol, (2) They had tried only once or twice out of curiosity, (3) Because of the stigma associated with alcohol consumption in girls.

Various literatures[26,27,31,32] have cited the influence of family members on a person's drinking with family history of 
alcohol consumption leading to increased likelihood of drinking. This is supported by the present study, which noted that $44.7 \%$ of students who were using alcohol had fathers with history of alcohol consumption. On the other hand, $13.3 \%$ of students who were not drinking alcohol had fathers with history of alcohol consumption. As shown in Table No. 8 and 9, similar trend was seen in mothers, brothers and sisters.

We also found a strong positive correlation between alcohol use and depression ( $\mathrm{p}$ value $<0.001$; R 0.415), which was similar to a finding by Sherina et al.[33]

As this was a cross-sectional study, conclusions about the direction of observed relationships cannot be drawn. Anonymity served as both strength as well as a limitation in our study. As strict anonymity was offered, students had no hesitation in filling up the questionnaires. On the other hand as the students were anonymous and could not be identified, those who needed help e.g. those with suicidal thoughts, those with AUDIT score of $>20$, etc. could not be given the help they needed.

\section{CONCLUSION}

This study suggests that depression and alcohol use are highly prevalent in undergraduate medical students. Since there is proof that depression or alcohol use during undergraduate medical training foresees future predicaments in physicians, effective measures must be taken up by concerned authorities. Medical students would benefit from greater assurance that the use of mental health services is confidential and completely separate from academic records.

\section{REFERENCES}

[1] Yusoff M, Rahim AAF, Baba AA, et al. The impact of medical education on psychological health of students: a cohort study. Psychology, Health \& Medicine 2013;18(4):420-30.

[2] Lindeman S, Laara E, Hakko $\mathrm{H}$, et al. A systematic review on gender-specific suicide mortality in medical doctors. Br J Psychiatry 1996;168(3):274-9.

[3] Lim M. Physician depression and suicide. Virtual Mentor 2003;5(9).

[4] Biswas T. First year MBBS student at AIIMS hospital found hanging in girls' hostel. NDTV.com 2017.https://www.ndtv.com/delhi-news/mbbsstudent-at-delhis-aiims-hospital-found-hanging-ingirls-hostel-1212380

[5] MBBS student commits suicide. The Hans India. 2017. http://www.thehansindia.com/posts/index/Crime/2 017-08-25/MBBS-student-commits-suicide/321619

[6] MBBS student jumps off fourth floor, dies. The Hindu, 2017. http://www.thehindu.com/todayspaper/mbbs-student-jumps-off-fourth-floordies/article18381187.ece

[7] Pradesh A. Service I. Medical student ends life by jumping off college building in Andhra Pradesh. NDTV.com 2017. https://www.ndtv.com/andhrapradesh-news/medical-student-ends-life-by-jumpingoff-college-building-in-andhra-pradesh-1632755

[8] Givens JL, Tjia J. Depressed medical students' use of mental health services and barriers to use. Academic Medicine 2002;77(9):918-21.
[9] Seshadri S. Substance abuse among medical students and doctors: a call for action. Natl Med J India 2008;21(2):57-9.

[10] Sohail N. Stress and academic performance among medical students. J Coll Physicians Surg Pak 2013;23(1):67-71.

[11] Beck AT, Ward CH, Mendelson M, et al. An inventory for measuring depression. Archives of General Psychiatry 1961;4(6):561-71.

[12] Babor T, Higgins-Biddle JC, Saunders JB, et al. AUDIT, the alcohol use disorders identification test. Dept. of Mental Health and Substance Dependence, 2nd edn. Geneva, Switzerland: World Health Organization 2001.

[13] Basnet B, Jaiswal M, Adhikari B, et al. Depression among undergraduate medical students. Kathmandu Univ Med J 2012;10(39):56-9.

[14] Kumar GS, Jain A, Hegde S. Prevalence of depression and its associated factors using Beck Depression Inventory among students of a medical college in Karnataka. Indian J Psychiatry 2012;54(3):223-6.

[15] Yadav R, Gupta S, Malhotra AK. A cross sectional study on depression, anxiety and their associated factors among medical students in Jhansi, Uttar Pradesh, India. Int $\mathrm{J}$ Community Med Public Health 2016;3(5):1209-14.

[16] Yusoff MSB, Rahim AAF, Baba AA, et al. Prevalence and associated factors of stress, anxiety and depression among prospective medical students. Asian J Psychiatry 2013;6(2):128-33.

[17] Qamar K, Khan NS, Kiani BMR. Factors associated with stress among medical students. J Pak Med Assoc 2015;65(7):753-5.

[18] Fares J, Al Tabosh H, Saadeddin Z, et al. Stress, burnout and coping strategies in preclinical medical students. North Am J Med Sci 2016;8(2):75-81.

[19] Altaf M, Altaf KF, Zahid S, et al. Medical students bearing mental stress due to their academic schedule. International Journal of Endorsing Health Science Research 2013;1(2):93-7.

[20] Mphele SBM, Gralewski C, Balogun S. Stress and alcohol use among college students: a case of Molepolole college students. IOSR Journal of Humanities and Social Science 2013;8(3):1-6.

[21] Fares J, Saadeddin Z, Al Tabosh H, et al. Extracurricular activities associated with stress and burnout in preclinical medical students. J Epidemiol Glob Health 2016;6(3):177-85.

[22] Maslach C, Schaufeli WB, Leiter MP. Job burnout. Annu Rev Psychol 2001;52:397-422.

[23] Park CL, Adler NE. Coping style as a predictor of health and well-being across the first year of medical school. Health Psychol 2003;22(6):627-31.

[24] Lamberti M, Napolitano F, Napolitano P, et al. Prevalence of alcohol use disorders among undergraduate and post-graduate healthcare students in Italy. PLoS One 2017;12(4):e0175719.

[25] Nash LM, Daly MG, Kelly PJ, et al. Factors associated with psychiatric morbidity and hazardous alcohol use in Australian doctors. Med J Aust 2010;193(3):161-6. 
[26] Goel N, Khandelwal V, Pandya K, et al. Alcohol and tobacco use among undergraduate medical students in India: a multicentric cross-sectional study. Central Asian Journal of Global Health 2015;4(1):187.

[27] Devi V, Ping ATW, Ying TT, et al. Alcohol use, its predictors and academic performance among Malaysian students of a medical college in India. Education in Medical Journal 2013;5(4):e72-e6.

[28] Apoorva, Pillai AS, Nayanar A, et al. Risk factors and consequences of alcohol consumption among college students. NUJHS 2014;4(2):102-4.

[29] Leavy RL, Alexander DA. Perceptions of drinking problems among undergraduate students in the United States and Scotland. International Journal of the Addictions 1992;27(10):1179-85.
[30] Delk E, Meilman PW. Alcohol use among college students in Scotland compared with norms from the United States. Journal of American College Health 1996;44(6):274-81.

[31] Kumar P, Basu D. Substance abuse by medical students and doctors. J Indian Med Assoc 2000;98(8):447-52.

[32] Jagnany VK, Murarka S, Haider S, et al. Pattern of substance abuse among undergraduate students in a medical college hostel. Health and Population Perspectives 2008;31(3):212-9.

[33] Sherina MS, Rampal L, Kaneson N. Psychological stress among undergraduate medical students. Med J Malaysia 2004;59(2):207-11. 